\title{
Efficient Holmium-doped solid-state lasers pumped by a Tm-doped silica fiber laser
}

\author{
Deyuan Shen, Jayanta Sahu and W. Andrew Clarkson
}

Optoelectronics Research Centre, University of Southampton, Southampton, SO17 1BJ, United Kingdom

In-band pumping of Ho-doped solid-state lasers by a cladding-pumped Tm fiber laser is an attractive route to high output power and high pulse energy in the eyesafe two-micron spectral region. This approach combines the advantages of fiber lasers and crystal solid-state lasers with relative immunity from the effects of thermal loading, nonlinear loss processes (e.g. stimulated Brillouin scattering) and energy-transfer-upconversion. The use of a Tm-doped fiber laser as the pump source allows a great deal of flexibility, since the broad emission linewidth allows the wavelength to be tuned over a very wide range spanning the absorption lines of interest in Ho:YLF, Ho:YAG and many other Ho-doped crystals.

In this paper, we report efficient operation of Ho:YAG and Ho:YLF lasers pumped by a tunable Tm-doped silica fiber laser. The lasing wavelength of the Tm-doped fibre laser could be tuned over $150 \mathrm{~nm}$ from $\sim 1860$ to $2010 \mathrm{~nm}$ with a relatively narrow linewidth $(<0.5 \mathrm{~nm})$ and at output power levels in excess of $9 \mathrm{~W}$. Using a simple standing-wave cavity configuration, $>6.4 \mathrm{~W}$ of $\mathrm{TEM}_{\mathrm{oo}}$ output was obtained from a Ho:YAG laser at $2.1 \mu \mathrm{m}$ at the maximum incident pump power of $9.6 \mathrm{~W}$, corresponding to an optical-to-optical efficiency of $67 \%$, and the slope efficiency with respect to incident pump power was $80 \%$. By comparision, for a similar resonator design, $4.8 \mathrm{~W}$ of output at $2.07 \mu \mathrm{m}$ was generated from a Ho:YLF laser at an incident pump power of $9.4 \mathrm{~W}$, corresponding to an optical conversion efficiency of $51 \%$. Using a simple ring resonator geometry and an acousto-optic modulator to enforce unidirectional operation, we have obtained $3.7 \mathrm{~W}$ of single-longitudinal-mode output from a Ho:YAG laser. The prospects for further improvement in performance and higher output power will be discussed.

Key words: Tunable Tm:fiber laser, Single frequency, Ho:YAG, Ho:YLF 